\title{
An Effective Hamiltonian Approach to Quantum Random Walk
}

\author{
Debajyoti Sarkar, Niladri Paul*† \\ Inter University Centre for Astronomy and Astrophysics, Pune 411007, India \\ Kaushik Bhattacharya, Tarun Kanti Ghosh $\ddagger$ \\ Department of Physics, Indian Institute of Technology, Kanpur 208016, India
}

\begin{abstract}
In this article we present an effective Hamiltonian approach for Discrete Time Quantum Random Walk. A form of the Hamiltonian for one dimensional quantum walk has been prescribed, utilizing the fact that Hamiltonians are the generators of time translations. Then an attempt has been made to generalize the techniques to higher dimensions. We find that the Hamiltonian can be written as the sum of a Weyl Hamiltonian and a Dirac comb potential. The time evolution operator obtained from this prescribed Hamiltonian is in complete agreement with that of the standard approach. But in higher dimension we find that the time evolution operator is additive, instead of being multiplicative like that of Ref. [1]. We showed that in case of two-step walk, effectively the time evolution operator can have multiplicative form. In case of a square lattice, quantum walk has been studied computationally for different coins and the results for both the additive and the multiplicative approaches have been compared. Using the Graphene Hamiltonian the walk has been studied on a Graphene lattice and we conclude the preference of additive approach over the multiplicative one.
\end{abstract}

\section{Introduction}

Quantum walk (QW) is a formulation implementing quantum principles on the classical random walk problem. The classical version in one dimension can be described with a two state coin. As the particle reaches a lattice point, the coin is tossed and the particle moves towards either $+x$ or $-x$ direction based on the outcome of the toss. But unlike the classical, if we include quantum concept, the particle would be in a superposition of $+x$ and $-x$ state in general and the random walk acquires a new nature as described in Ref. [2], [3], [4], [5], [6] and others. Quantum walk on a graph with different coins has been studied in Ref. [7], [6], [8], [9] in great detail. Quantum random walk has turned out to be very useful in developing algorithms for quantum computation as studied in Ref. [10], [11], [12], [13], [14] and [15].

In Ref. [5],[4], the problem of quantum walk on a line was studied in Fourier space and an analytic solution was obtained in the asymptotic limit. The effect of absorbing boundaries was discussed in Ref. [5],[16]. In case of classical random walk, the distribution attains a Gaussian nature asymptotically in time with a central peak and the variance is proportional to time; whereas in case of quantum walk, the distribution spreads out from the centre and the variance is proportional to the square of time as discussed in Ref. [4], [6]. Quantum walk in two dimension has been discussed in case of square and triangular lattice with various coins like Hadamard, Grover, DFT etc. in Ref. [17], [18], [1] and others. In this case also the distribution of the probability of finding the particle spreads away from the centre in general.

In recent years, experiments have been performed to implement discrete time quantum random walk in case of NMR Quantum Information Processor cf. Ref. [19], [20], trapped atoms cf. Ref. [21], ions cf. Ref. [22], [23] and photons cf. Ref. [24], [25]. The use of Dirac-like Hamiltonian in the context

${ }^{*}$ Debajyoti Sarkar, Niladri Paul did a significant amount of work presented in this article when they were in the Department of Physics, Indian Institute of Technology, Kanpur 208016, India.

${ }^{\dagger}$ Email: debajyoti, npaul @iucaa.in

${ }^{\ddagger}$ kaushikb, tkghosh@iitk.ac.in 
of unitary quantum cellular automata was discussed in Ref. [26]. Recently the Hamiltonian of the Discrete Time Quantum Random Walk (DTQRW) has been studied in Ref. [1].

The material in the article is presented in the following way. In Sec. 2 we briefly introduce the concept of the quantum walk. In Sec. 3 we give a prescription to formulate the Hamiltonian of a QW system from a physical point of view and thereby obtain the time evolution operator from that Hamiltonian in Sec. 3.1. We extend our Hamiltonian approach for two dimensional lattice in Sec. 3.2 and it is found that the evolution operator is additive in nature instead of being multiplicative as mentioned in Ref. [1]. The other difference with Ref. [1] is the way in which the effective Hamiltonian is perceived. In the above reference the effective Hamiltonian does not have separate information about coin operation and lattice translation, where as in our approach the effective Hamiltonian shows a clear distinction between the two separate operations. If we take the evolution operator to be multiplicative, the walk becomes a two step walk; i.e. the particle must move along $Y$ direction after moving along $X$ direction and vice versa. Two consecutive moves along the same direction is forbidden. But in our approach, it becomes a single step walk, i.e. the particle is allowed to move along the same direction in two consecutive steps. No path is forbidden. This notion of two-step and single step walk has been discussed in detail in Sec. 3.2. In Sec. 3.2.2, we compare the results of both approach computationally in case of a square lattice using different quantum coins. In the Sec. 4, the quantum walk on a Graphene lattice has been studied starting from the Graphene Hamiltonian described in Ref. [27]. In this case also we have presented a comparative study of the additive and multiplicative approach.

\section{Concept of Quantum Walk}

In case of quantum walk, the particle can be in a superposition of the available basis coin states instead of a definite one as in the case of a classical random walk. In one dimension this is equivalent to a two level problem. The two basis states of the coin can be represented by the kets $|+\rangle$ and $|-\rangle$. We assume that $|+\rangle$ is a coin state for which the quantum particle moves in the $-x$ direction where as for the other coin state $|-\rangle$ the particle moves in the $+x$ direction. A general coin state can be represented as

$$
|\chi\rangle=c_{+}|+\rangle+c_{-}|-\rangle .
$$

As soon as the particle reaches any lattice point, the state of the coin is changed; e.g. if the coin state of the particle was in an eigenstate $(| \pm\rangle)$, it changes to a superposed state of them. To include superposition we need an operator which operates on $|+\rangle$ and $|-\rangle$ and results in a superposed state. Hadamard coin described in Ref. [4] is a commonly used mixing operator in this regard. The effect of Hadamard operator on a coin state can be described as

$$
\begin{aligned}
& H_{c}|+\rangle=\frac{1}{\sqrt{2}}(|+\rangle+|-\rangle), \\
& H_{c}|-\rangle=\frac{1}{\sqrt{2}}(|+\rangle-|-\rangle) .
\end{aligned}
$$

In the following section, we discuss the Hadamard walk in brief in position space formalism.

\subsection{Position Space Formulation}

Following Ref. [5] and [4], we consider the basis state of the particle as a product state of the chirality and the position basis states : $| \pm\rangle|x\rangle$. Therefore we can define a translation operator $T$ similar to that of Ref. [1], as

$$
T(n)=|+\rangle\langle+|\otimes| n-1\rangle\langle n|+|-\rangle\langle-|\otimes| n+1\rangle\langle n| ;
$$

where $n$ is the index of the lattice points. Here we have assumed that at each step the particle moves from one lattice point to the nearest lattice points only and the lattice spacing is unity. Now in a 
basis of $|+\rangle \otimes \mathbb{1}_{N}$ and $|-\rangle \otimes \mathbb{1}_{N}, T(n)$ has the form,

$$
T(n)=\left(\begin{array}{cc}
|n-1\rangle\langle n| & 0 \\
0 & |n+1\rangle\langle n|
\end{array}\right) .
$$

Here, $\mathbb{1}_{N}{ }^{1}$ is the identity matrix in $N$ dimension whereas $N$ denotes the total number of discrete lattice points. Hence the time evolution operator is given by

$$
\begin{aligned}
W(n) & =T(n) H_{c} \\
& =\frac{1}{\sqrt{2}}\left(\begin{array}{cc}
|n-1\rangle\langle n| & |n-1\rangle\langle n| \\
|n+1\rangle\langle n| & -|n+1\rangle\langle n|
\end{array}\right) .
\end{aligned}
$$

Here $H_{c}$ is the Hadamard coin. In general, in place of $H_{c}$, we can use any other quantum coin. The above one is the expression of the time evolution operator if the particle was at the $n$-th lattice point. Since the particle could be in any of the lattice points, the total time evolution operator will be

$$
\mathbb{W}=\sum_{n} W(n)
$$

This is the time evolution operator for 1-D walk in case of position space formulation.

\section{Hamiltonian Method}

In this section we develop the Hamiltonian formulation of quantum walk. In case of quantum walk the time evolution operator arises from two processes. First at the lattice points, the chiral state of the particle changes and for that, at a given lattice point $n$, one can write the chirality flipping operator as $W_{1}(n)=S \otimes|n\rangle\langle n|$. Here $S$ is a $2 \times 2$ unitary operator. Then one uses a translational operator $T(x)$ which moves the particle from one position to another position in space. Clearly, $T(x)$ is a continuous operator acting at any point $x$ in space (not necessarily lattice points). In case of the lattice points, the time evolution operator for a single step is given by, $W(n)=T(n) W_{1}(n)$. At places other than the lattice points $T(x)$ can itself act as the time evolution operator since at those places, the chiral state of the particle remains unchanged. This perception of $W(n)$ immediately leads to the concept of an effective Hamiltonian for the system which acts as the generator for time evolution. Like any other traditional Hamiltonian this effective Hamiltonian should be Hermitian.

Since the one dimensional quantum walk problem is a two level problem, we expect the Hamiltonian to be proportional to a $2 \times 2$ matrix. We expect the wave function of the particle to be just translated in between two lattice points remaining unchanged in its form. Hence we consider the Hamiltonian proportional to $k v$ where $p=\hbar k$ and $v$ is the effective velocity of the quantum particle, not to be confused with the Lagrangian velocity. A straightforward calculation shows that when the Hamiltonian $k v$ operates on some wave function, it just translates the state keeping the form of wave function invariant. Hence the Hamiltonian representing translation must be proportional to $k v$. So we expect the Hamiltonian to be of the form $\alpha k v \otimes \mathbb{1}$ in between two lattice points and $\beta \otimes|n\rangle\langle n|+\alpha k v \otimes \mathbb{1}$ on the lattice points. Here, $|n\rangle\langle n|$ is used to make sure that $\beta$ acts only at the lattice points. The term $\alpha k v$ is valid at all positions in space (even outside the lattice points) and hence we get the term $\mathbb{1}$. Here both $\alpha$ and $\beta$ are $2 \times 2$ Hermitian matrices. Now we can write the translational operator $T(x)$ (which displaces the particle from a point $x$ to $x \pm \Delta x, \Delta x$ being the separation between two points in space, not necessarily the separation between two lattice points) as

$$
T(x)=\left(\begin{array}{cc}
|x-\Delta x\rangle\langle x| & 0 \\
0 & |x+\Delta x\rangle\langle x|
\end{array}\right)
$$

\footnotetext{
${ }^{1}$ In our convention, $\mathbb{1}_{n}$ (where $n \in N$, the set of natural numbers) denotes $n \times n$ identity operator in finite dimensional space, $\mathbb{1}$ denotes infinite dimensional identity operator acting on the space spanned by the coordinates of all the points in space and $\mathcal{I}$ denotes infinite dimensional identity operator such that $\mathcal{I}=\mathbb{1}_{n} \otimes \mathbb{1}$
} 
If we denote the Hamiltonian corresponding to the translation operator $T$ as $H_{T} \otimes \mathbb{1}$, then following Appx. A.1,

$$
H_{T} \otimes \mathbb{1}=-k v \sigma_{z} .
$$

In deriving the previous expression, we have taken $\hbar=1$ and have defined $v \equiv \frac{\Delta x}{\Delta t}$ as the effective speed of the quantum particle. Throughout this article, we will always consider $\hbar=1$. The derived Hamiltonian $H_{T} \otimes \mathbb{1}$ is valid at all positions, whether the position is a lattice point or not.

If the Hamiltonian corresponding to the coin operation, $W_{1}(n)$, is denoted by $H_{S} \otimes|n\rangle\langle n|$, following Appx. A.2, we can write,

$$
H_{S}=\frac{i}{\Delta \tau} \ln S
$$

Here, $\Delta \tau$ is the time interval to flip the spin, i.e. it is the time interval the particle stays at the lattice point $n$. It is assumed that the particle stays for a very short time-interval at the lattice point $n$, hence $\Delta \tau$ is much smaller compared to the time scale in which the particle is translated from one lattice point to the other. We can write the total Hamiltonian as

$$
H=H_{T} \otimes \mathbb{1}+\sum_{n} H_{S} \otimes|n\rangle\langle n| .
$$

Therefore, following Appx. A.3, for any point $x$ the position space representation of the Hamiltonian is given by

$$
H(x)=H_{T}+\sum_{n} H_{s} \delta(x-n) .
$$

The Hamiltonian has a continuous term $-\sigma_{z} k v$ and a Dirac comb term. This can be interpreted as Weyl Hamiltonian with a Dirac comb potential ${ }^{2}$

\subsection{Time evolution operator from a Dirac like Hamiltonian in $1 \mathrm{D}$}

\subsubsection{Formulation and numerical simulation}

Let's consider the generalized Hamiltonian as derived in Eq.(10). Therefore, we can write our time evolution operator at the $n$-th lattice point as

$$
\begin{aligned}
W(n, \Delta t) & =e^{-i H_{T} \Delta t \otimes \mathbb{1}} e^{-i H_{S} \Delta \tau \otimes|n\rangle\langle n|} \\
& =T(n)(S \otimes|n\rangle\langle n|) .
\end{aligned}
$$

We can write down the time evolution operator here as the product of the two terms in this way because the term $\Delta t\left[H_{S}, H_{T}\right] \otimes|n\rangle\langle n|$ goes to zero due to the following reason. When the particle is at some lattice point for the time interval $\Delta \tau, \Delta x \rightarrow 0$ and $\lim _{\Delta x \rightarrow 0} H_{T} \Delta \tau=0$. Further, when the particle is not at a lattice point, $H_{S}=0$ by our formulation. So we get rid of the commutation term. Physically we can say that the particle would stay at a lattice point $n$ for an infinitesimal time interval $\Delta \tau$. In that time interval, the effect of $H_{T}$ can be neglected. Equivalently, we can say that during the chirality flip, the translation is almost zero. Therefore the effect of $H_{T} \Delta \tau$ can be neglected. The particle takes some finite time $\Delta t$ to reach the next lattice point and when it is in between two lattice points, $H_{S}$ does not have any effect on its state. Hence we can consider the action of the time evolution operator for a particular lattice point for a finite time interval $\Delta t$, as an operation to first mix the coin states of the particle within an infinitesimal time interval $\Delta \tau$, followed by a translation occurring in a time interval $\Delta t-\Delta \tau$ towards a particular direction depending on its chirality. While deriving Eq.(12), at the final step we have assumed that $\Delta x=\Delta t=1$.

In general, for a $S O(2)$ matrix $S=\left(\begin{array}{cc}\cos \theta & -\sin \theta \\ \sin \theta & \cos \theta\end{array}\right)$, we get the spin Hamiltonian, $H_{S}=\frac{\theta}{\Delta \tau} \sigma_{y}$.

\footnotetext{
${ }^{2}$ At a first glance, Eq.(11) may look like dimensionally inconsistent but it is not, since while converting a functional from its discrete to continuous form we must divide the discrete form with a weight factor of dimension same as that of the continuous variable. In this expression we have used a weight factor of unit length.
} 


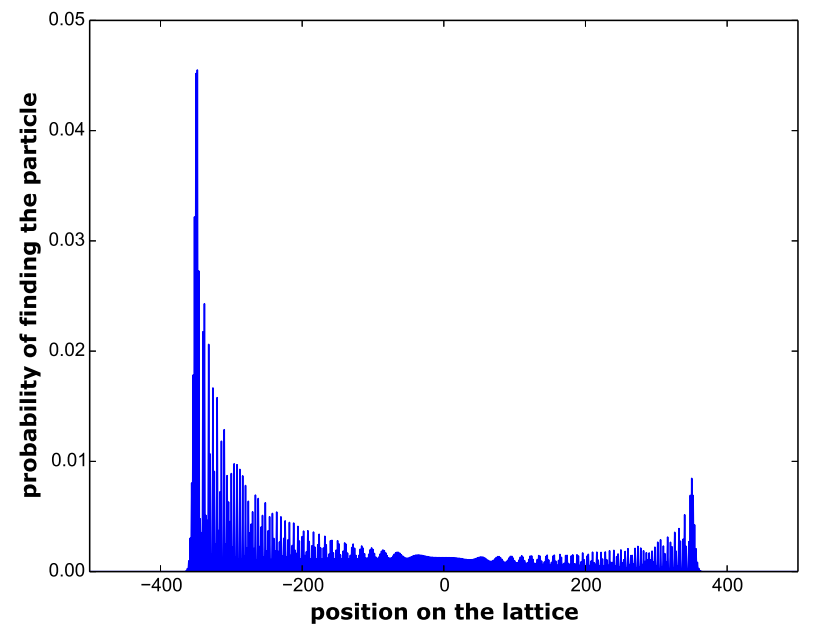

Figure 1: In this figure the particle started quantum walk from the lattice point 0 . After 500 steps probability distribution of the outcome is shown in the figure.

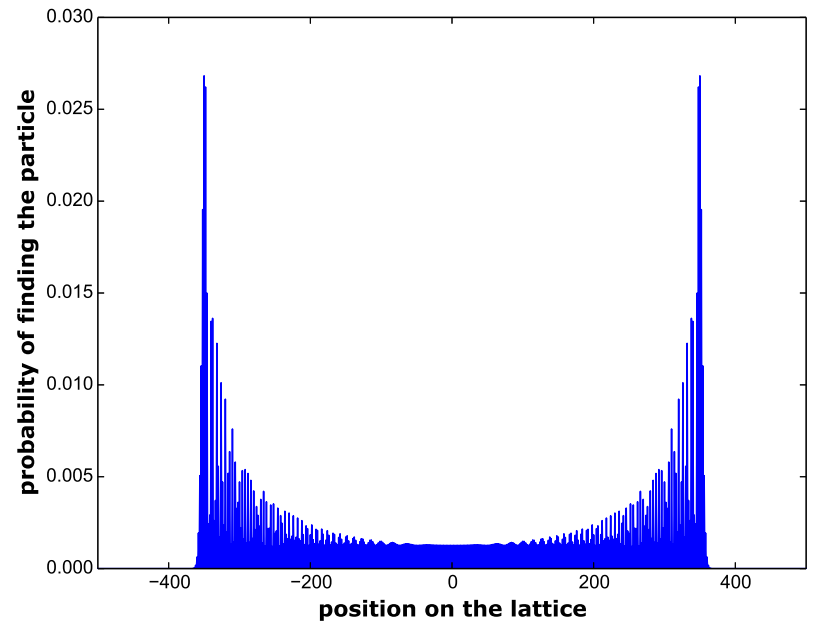

Figure 2: In this figure the particle started quantum walk from the lattice point 0 . After 500 steps probability distribution of the outcome is shown in the figure.

For a $S U(2)$ matrix $S=\left(\begin{array}{cc}\cos \theta & i \sin \theta \\ i \sin \theta & \cos \theta\end{array}\right)$, we have $H_{S}=-\frac{\theta}{\Delta \tau} \sigma_{x}$. If we choose $\theta=\frac{\pi}{4}$ in the previous expression, then we get, $S=Y=\frac{1}{\sqrt{2}}\left(\begin{array}{cc}1 & i \\ i & 1\end{array}\right)$ and thereby get $H_{S}=-\frac{\pi}{4 \Delta \tau} \sigma_{x}$. The result of 1-D quantum walk for this coin is shown in Fig.2. If we choose the Hadamard operator as $S=H_{c}=$ $\frac{1}{\sqrt{2}}\left(\begin{array}{cc}1 & 1 \\ 1 & -1\end{array}\right)$, then the spin Hamiltonian $H_{S}=\frac{\pi}{2 \Delta \tau}\left(\begin{array}{cc}-1+\frac{1}{\sqrt{2}} & \frac{1}{\sqrt{2}} \\ \frac{1}{\sqrt{2}} & -1-\frac{1}{\sqrt{2}}\end{array}\right)$. The result of quantum walk with the Hadamard coin is shown in Fig.1. For both Fig.1 and Fig. 2, we have chosen our initial chiral state to be $|\chi\rangle=\frac{1}{\sqrt{2}}(|+\rangle+|-\rangle)$. The particle starts at the middle position of the lattice points, so its initial position state is $|\xi\rangle=|0\rangle$. Therefore, the total initial state of the particle $|\psi\rangle=|\chi\rangle \otimes|\xi\rangle$. The results of Fig. 1 and Fig. 2 are the standard results of one dimensional quantum walk well studied in the previous literatures. In rest of the cases of this article, we will generally choose that the particle starts from the middle point of the lattice structure unless otherwise stated. So in general, only the initial chiral states will be mentioned. Always the initial state of the particle is chosen to be a direct product state of the coin state and the position state.

\subsubsection{Calculation of variance}

We calculated the variance of this 1-D walk for different no. of steps numerically. The result shown in the Fig.3 is for the Y coin and an initial chiral state $|\chi\rangle=\frac{1}{\sqrt{2}}(|+\rangle+|-\rangle)$. The plot can be approximated as a polynomial of the form $0.47175578+0.00091395 t+0.29289026 t^{2}$. Clearly the leading order term in the variance $\sim t^{2}$, unlike the classical walk as already pointed out in Ref. [6] and others. 


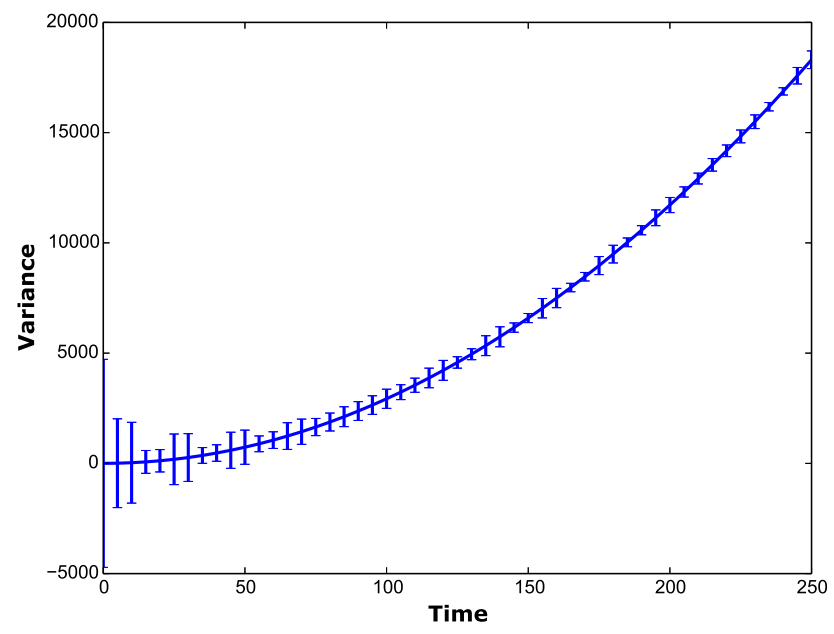

Figure 3: Plot of variance vs. time in case of $1 \mathrm{D}$ quantum walk. The errorbar is with respect to the quadratic polynomial is multiplied by $10^{4}$. The $\mathrm{Y}$ coin has been used to generate the plot.

\subsection{Two Dimensional case for a square lattice}

In Ref. [1], while formulating random walk on a square lattice, the Hamiltonian has been taken in additive form and the total time evolution operator has been taken as the product of the evolution operator acting along each axis. It implies that the particle has to move in $x-y$ or $y-x$ order only. It does not have the choice to move along the same axis in two consecutive steps. This approach can be thought of taking two steps (in $x-y$ or $y-x$ order only) in each operation of the evolution operator. So we call it two step approach in the following sections of this article. But in our approach, the particle is allowed to move along any axis in each step. Hence in this single step approach there is no forbidden path for the walk.

\subsubsection{Formulation}

In random walk, a particle can move along any of the paths available to it from a lattice point. So in case of quantum walk the dimensionality of the coin (i.e. the dimension of the Hilbert space on which the coin acts) is determined by the number of paths the particle can choose. In all dimensions higher than one, we have two types of chirality of the particle for the approach described in this article. One is to choose which axis the particle would move along in the next step and the other to decide whether the particle would go to the positive or to the negative direction of the chosen axis.

In case of a square lattice, the particle has two choice of axes at each lattice point. In this case, analogous to Eq. (11), we can say that the Hamiltonian at a point $(x, y)$ for 2 -D walk will have the form

$$
H(x, y)=\sum_{n, m} H_{s} \delta(x-n) \delta(y-m)+H_{T} \otimes \mathbb{1} .
$$

Here,

$$
\begin{array}{r}
H_{S}=\frac{i}{\Delta \tau} \ln \left(S_{x} \otimes M_{1}+S_{y} \otimes M_{2}\right), \\
H_{T}=-\left(k_{x} v_{x}\left(\begin{array}{ll}
1 & 0 \\
0 & 0
\end{array}\right)+k_{y} v_{y}\left(\begin{array}{ll}
0 & 0 \\
0 & 1
\end{array}\right)\right) \otimes \sigma_{z} .
\end{array}
$$

Where $S_{x}$ and $S_{y}$ are given as,

$$
S_{x}=\left(\begin{array}{cc}
S_{11} & S_{12} \\
0 & 0
\end{array}\right) \quad \text { and } \quad S_{y}=\left(\begin{array}{cc}
0 & 0 \\
S_{21} & S_{22}
\end{array}\right) .
$$


We define $S=S_{x}+S_{y}$ as the chirality operator for choosing the $X$ or the $Y$ axis. $M_{1}$ and $M_{2}$ are chirality operators for choosing positive or negative direction of a particular axis. Now, if we choose discrete set of lattice points such that the position eigenstate is $|n, m\rangle$, with the condition $\langle n, m \mid p, q\rangle=\delta_{n p} \delta_{m q}(n, m, p, q$ are integers), then following similar approach as shown in Sec. 3.1.1, we can write (Appx. B),

$$
W_{n m}=T_{n m}\left(\left(S_{x} \otimes M_{1}+S_{y} \otimes M_{2}\right) \otimes|n, m\rangle\langle n, m|\right),
$$

where

$$
T_{n m}=\left(\begin{array}{cccc}
|n-1, m\rangle\langle n, m| & 0 & 0 & 0 \\
0 & |n+1, m\rangle\langle n, m| & 0 & 0 \\
0 & 0 & |n, m-1\rangle\langle n, m| & 0 \\
0 & 0 & 0 & |n, m+1\rangle\langle n, m|
\end{array}\right)
$$

is the translation operator. The result of Eq.(17) shows that the time evolution operator is additive in nature. This clearly differs from the two-step ${ }^{3}$ approach of Ref. [1] where it is assumed that the two dimensional time evolution operator is the product of one dimensional time evolution operator in the $X$ and $Y$ direction. Now for the total chirality operator $\mathbb{M}=S_{x} \otimes M_{1}+S_{y} \otimes M_{2}$ we can use different types of mixing operators, e.g. Grover, DFT or the higher dimensional Hadamard coin as discussed in the next section.

\subsubsection{Plots for different coins}

Case 1 : First let's choose the coin as the direct product of two Hadamard coins.
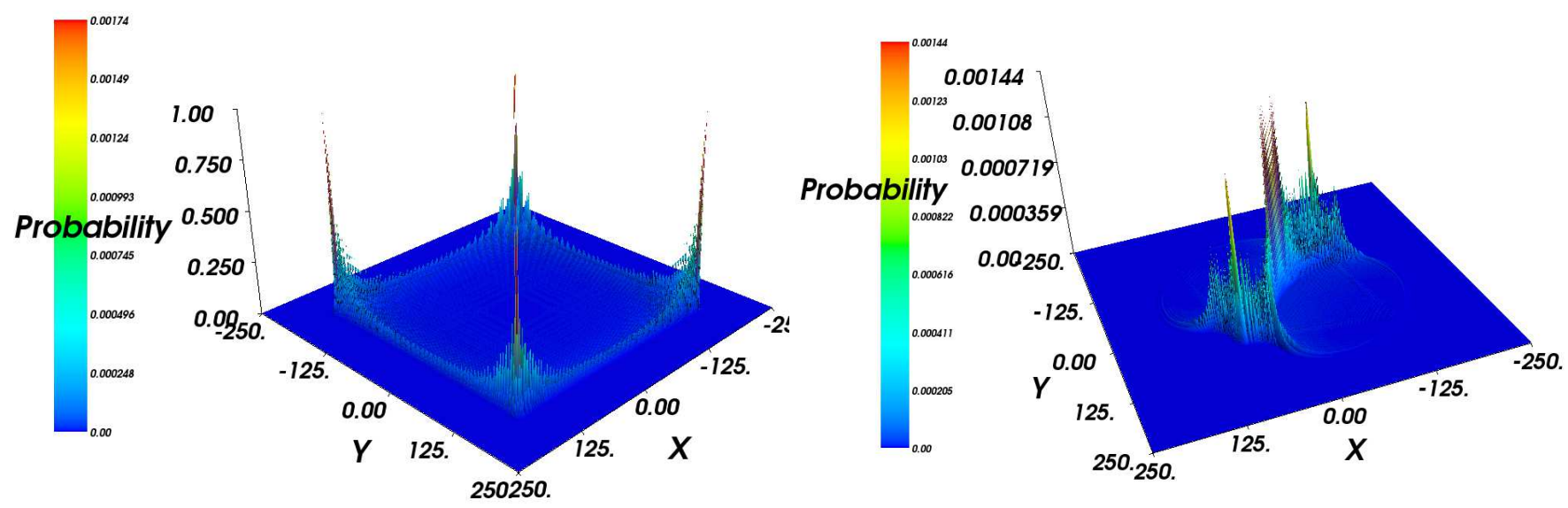

Figure 4: Probability of finding the particle after 250 steps starting from the origin. This plot is with the two-step approach using the coin of Eq.(18).
Figure 5: Probability of finding the particle after 250 steps starting from the origin. This plot is with the one-step approach using the coin of Eq.(18)

In that case,

$$
\mathbb{M}=\frac{1}{2}\left(\begin{array}{cccc}
1 & 1 & 1 & 1 \\
1 & -1 & 1 & -1 \\
1 & 1 & -1 & -1 \\
1 & -1 & -1 & 1
\end{array}\right)
$$

\footnotetext{
${ }^{3}$ In the two-step approach if the particle moves along $x$ axis at one step, the next step must be along $y$-axis. So we can interpret the method as follows: first $W_{x}$ operates on the particle and displaces it along $x$-direction. When it reaches to the next lattice point, $W_{y}$ operates. So in this case the time evolution operator is different at different lattice points. The total time evolution operator for the lattice is the sum of the time evolution operator at all the lattice points. If the particle starts to move along $X$ direction from the point $(0,0)$, at all the even points (points for which $x+y$ is even) the operator $W_{x}$ will act while at all the odd points (points for which $x+y$ is odd) the operator $W_{y}$ will act. Although the operator is not actually multiplicative, the net effect in two-step can be described by $W_{y} W_{x}$ because after evolution by $W_{x}$ the particle would reach next lattice point where the evolution is governed by $W_{y}$. This can happen if at the points where $W_{x}$ is operating, $k_{y} v_{y}=0$ and at the points where $W_{y}$ is operating, $k_{x} v_{x}=0$. In this way, we can reproduce the two-step approach of Ref. [1] starting from our Hamiltonian formulation of single-step walk.
} 
We choose our initial chiral state to be $|\chi\rangle=\frac{1}{\sqrt{2}}(|1\rangle+i|2\rangle) \otimes \frac{1}{\sqrt{2}}(|+\rangle+i|-\rangle)$. From now onwards $|1\rangle,|2\rangle$ will denote the chiral state of the particle to choose the $X$ and the $Y$ axis respectively and $|+\rangle,|-\rangle$ denote whether the particle will move along the positive or negative side of a chosen axis respectively. The result of the walk governed by this coin is shown in Fig.4 and Fig. 5. The spin Hamiltonian for this coin is given as

$$
H_{S}=\frac{\pi}{4 \Delta \tau}\left(\begin{array}{cccc}
-1 & 1 & 1 & 1 \\
1 & -3 & 1 & -1 \\
1 & 1 & -3 & -1 \\
1 & -1 & -1 & -1
\end{array}\right)
$$

Case 2 : Now we choose the Grover coin described in Ref. [6]. Therefore,

$$
\mathbb{M}=\frac{1}{2}\left(\begin{array}{cccc}
-1 & 1 & 1 & 1 \\
1 & -1 & 1 & 1 \\
1 & 1 & -1 & 1 \\
1 & 1 & 1 & -1
\end{array}\right)
$$

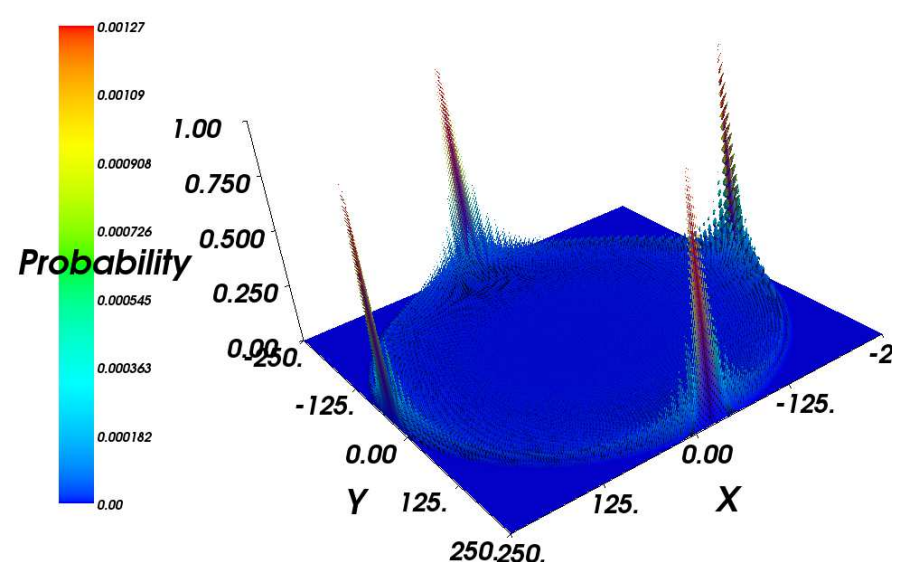

Figure 6: Probability of finding the particle after 250 steps starting from the origin. This plot is with the two-step approach using the Grover coin of Eq.(20).

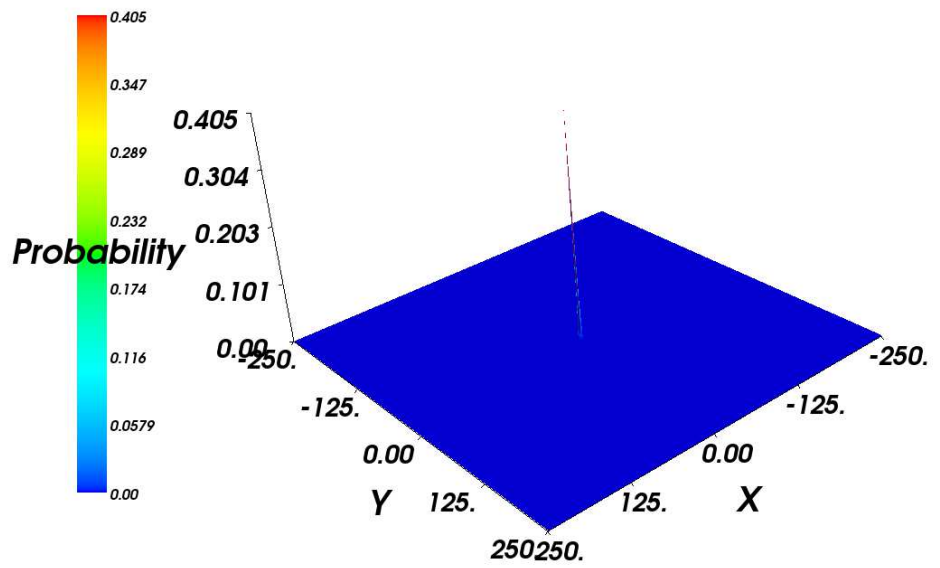

Figure 7: Probability of finding the particle after 250 steps starting from the origin. This plot is with the one-step approach using the Grover coin of Eq.(20).

and we choose our initial chiral state as $|\chi\rangle=\frac{1}{\sqrt{2}}(|1\rangle-|2\rangle) \otimes \frac{1}{\sqrt{2}}(|+\rangle-|-\rangle)$. The result is as shown in Fig. 6 and Fig. 7. The spin Hamiltonian $H_{S}$ for this coin is given by,

$$
H_{S}=\frac{\pi}{4 \Delta \tau}\left(\begin{array}{cccc}
-3 & 1 & 1 & 1 \\
1 & -3 & 1 & 1 \\
1 & 1 & -3 & 1 \\
1 & 1 & 1 & -3
\end{array}\right)
$$

Case 3 : Now we choose the DFT coin described in Ref. [6]. Therefore,

$$
\mathbb{M}=\frac{1}{2}\left(\begin{array}{cccc}
1 & 1 & 1 & 1 \\
1 & i & -1 & -i \\
1 & -1 & 1 & -1 \\
1 & -i & -1 & i
\end{array}\right) \quad(\text { where, } i=\sqrt{-1} \text { ) }
$$




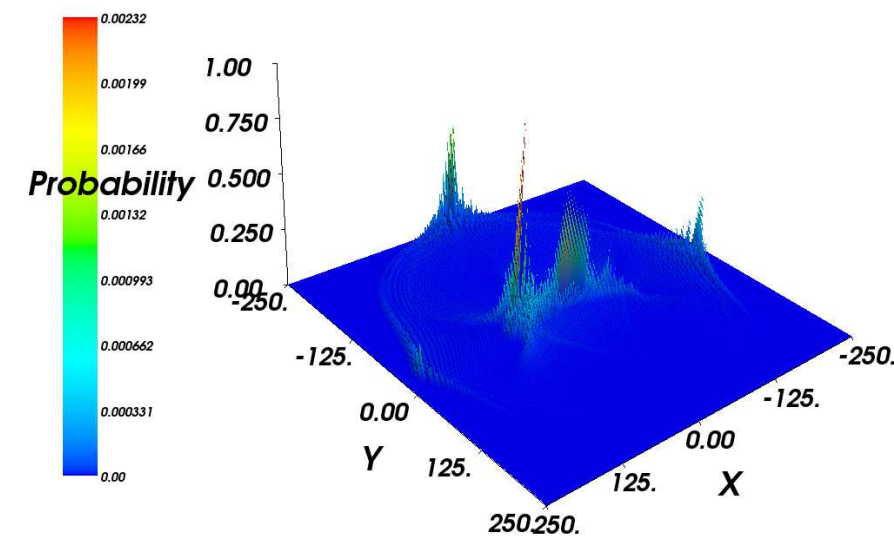

Figure 8: Probability of finding the particle after 250 steps starting from the origin. This plot is with the two-step approach using the DFT coin of Eq.(22).

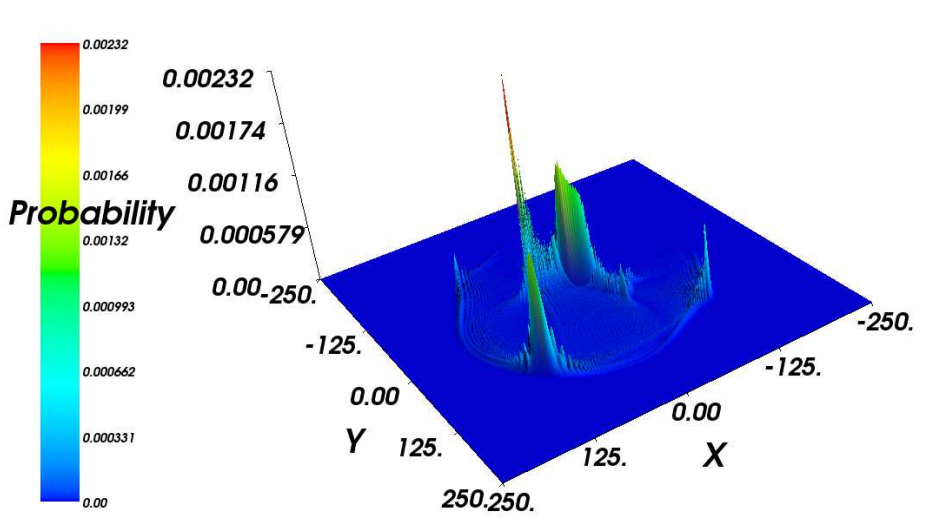

Figure 9: Probability of finding the particle after 250 steps starting from the origin. This plot is with the one-step approach using the DFT coin of Eq.(22).

and we choose our initial chiral state $|\chi\rangle=\frac{1}{\sqrt{2}}(|1\rangle-|2\rangle) \otimes \frac{1}{\sqrt{2}}(|+\rangle-|-\rangle)$. The result is as shown in Fig.8 and 9. The spin Hamiltonian for this coin is given by

$$
H_{S}=\frac{\pi}{4 \Delta \tau}\left(\begin{array}{cccc}
-1 & 1 & 1 & 1 \\
1 & -2 & -1 & 0 \\
1 & -1 & -1 & -1 \\
1 & 0 & -1 & -2
\end{array}\right)
$$

The plots of Fig. 4, 6 and 8 are the standard two-step quantum walks well studied before in the other literatures but they have been shown here to compare the results with that of the single-step approach of the corresponding coins.

\subsubsection{Calculation of variance}

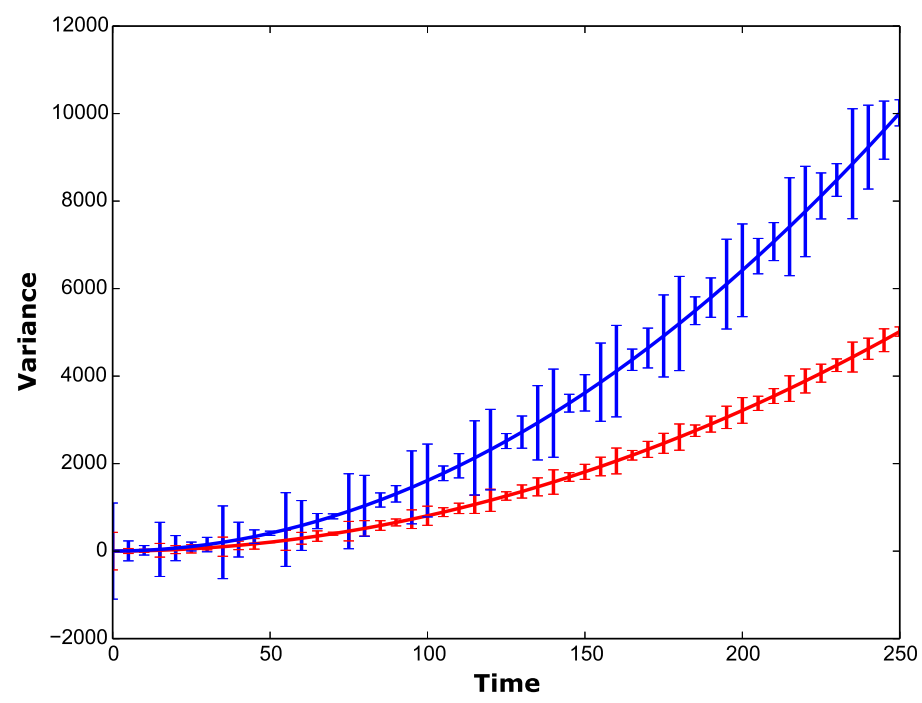

Figure 10: Plot of variance with time in case of a square lattice. The red curve shows variance in case of the single step approach and the blue one for two step approach. The errorbar with respect to the quadratic polynomial in both cases is multiplied by a factor of $10^{3}$. The DFT coin of Eq.(22) has been used to generate the plot. 
We calculated the variance of the probability distribution for the square lattice case and result has almost similar form with all types of coins we have used. So we mention here only the result obtained with the DFT coin. The above figure is obtained with an initial chiral state, $|\chi\rangle=\frac{1}{\sqrt{2}}(|1\rangle+|2\rangle) \otimes$ $\frac{1}{\sqrt{2}}(|+\rangle+|-\rangle)$. The red color plot in Fig.10 is for single step approach and can be approximated with an approximated polynomial of the form $0.42904373+0.08807425 t+0.07993539 t^{2}$ and the blue color plot is for two step approach and can be described with an approximated polynomial $1.09843965+$ $0.17597539 t+0.15954993 t^{2}$. So we see that the variance of the probability distribution in QRW has quadratic nature with time in case of $2 \mathrm{D}$ square lattice, similar to the form for $1 \mathrm{D}$ case as mentioned in Ref. [4], [6].

\section{Quantum walk on Graphene Lattice}

The Graphene Hamiltonian according to Ref. [27] is given by $H=v_{F} \vec{\sigma} \cdot \vec{p}$ which is valid only at positions very close to a lattice point. Taking $\hbar v_{F}=1$, we can write $H=\vec{\sigma} \cdot \vec{k}=\sigma_{x} k_{x}+\sigma_{y} k_{y}$. We will see that this Hamiltonian can generate an infinitesimal time evolution operator close to a lattice point. To generate a finite time evolution operator we need the Hamiltonian for translation operator to act at positions in between two lattice points.

\subsection{Formulation of the problem}

To describe random walk problem on Graphene lattice, we have to consider the three available paths at each point. So we need three axes as defined by $n_{1}, n_{2}$ and $n_{3}$ as shown in the Fig.11. We denote the directions along these three axes by 1,2 and 3 respectively. Clearly these three quantities $n_{1}, n_{2}, n_{3}$ are not linearly independent but they are dependent by the relations

$$
x=\frac{\sqrt{3}\left(n_{2}-n_{3}\right)}{2} \text { and } y=-n_{1}+\frac{\left(n_{2}+n_{3}\right)}{2} .
$$

By choosing these three axes we can easily extend our previous approach for square lattice in this case. In this approach, the position ket at each lattice point $(x, y)$ is denoted by $\left|n_{1}, n_{2}, n_{3}\right\rangle$. For Graphene

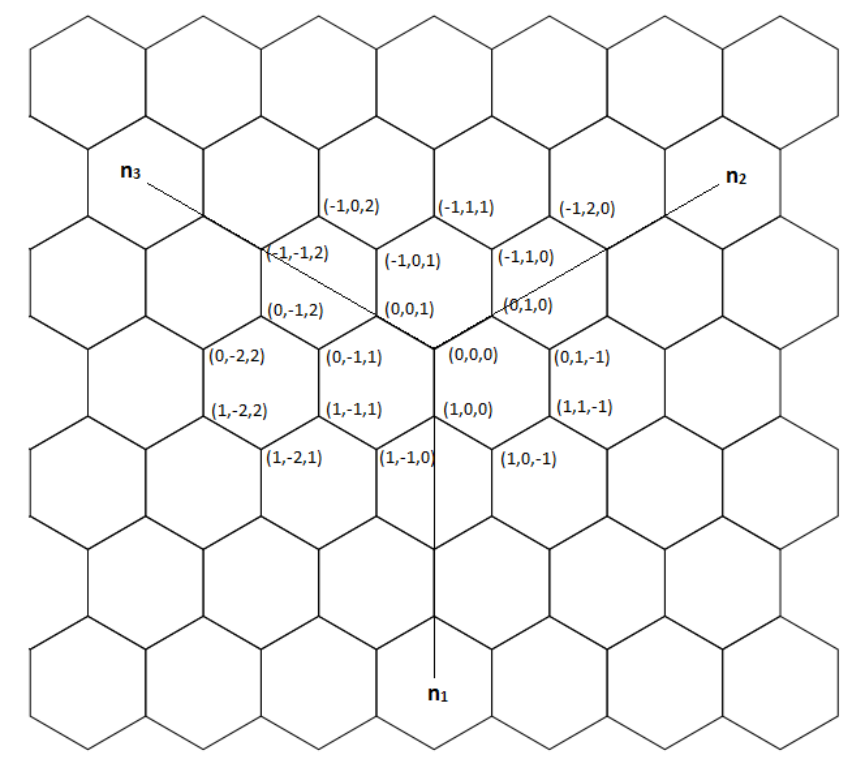

Figure 11: Here Graphene lattice is presented with the three axes $n_{1}, n_{2}$ and $n_{3}$. Some of the lattice points are specified on the lattice.

lattice, we can classify the lattice points into two types. The points for which the sum of $n_{1}, n_{2}$ and $n_{3}$ is even, are called even points and those having an odd sum of lattice indices are classified as odd 
points. Let us consider the two nearby lattice points, say the even point $(0,0,0)$ denoted by point 1 and the odd point $(0,1,0)$ denoted by point 2 . From the point 1 , the particle can move only to the positive direction of all the three axes (see Fig.11). But from the point 2, the particle moves only along the negative direction of all the three axes. Clearly, the geometry itself constraints the direction of movement here. We can describe this by using two kets as $|+\rangle$ and $|-\rangle$ which denotes the chirality of going along the negative or positive direction respectively as in the earlier cases. The only difference in this case is that the particle cannot be at superposition of $|+\rangle$ and $|-\rangle$, it must be at either of them. It is clear from the geometry that if the particle is in $| \pm\rangle$ state at some step, at the next step it must be in $|\mp\rangle$ state. For the lattice as described in Fig. 11, for even points, the particle is always in $|-\rangle$ state and for the odd points it is always in $|+\rangle$ state as per our convention. Clearly the operator needed to describe this kind of motion is given by $\left(\begin{array}{ll}0 & a \\ b & 0\end{array}\right)$. Again, we need a $3 \times 3$ matrix to make the superposition of three chiral states which determine the choice one of the three axes. After choosing the chirality, the state must translate. Following Eq.(17), we can write the general time evolution operator as $W\left(n_{1}, n_{2}, n_{3}\right)=T\left(n_{1}, n_{2}, n_{3}\right)\left(\left(S_{1} \otimes M_{1}+S_{2} \otimes M_{2}+S_{3} \otimes M_{3}\right) \otimes\left|n_{1}, n_{2}, n_{3}\right\rangle\left\langle n_{1}, n_{2}, n_{3}\right|\right)$. Here $S_{1}, S_{2}$ and $S_{3}$ are $3 \times 3$ matrices which have only the $1^{\text {st }}, 2^{\text {nd }}$ and $3^{\text {rd }}$ rows non-zero respectively. We can choose any three dimensional unitary operator as $S$ (where, $S=S_{1}+S_{2}+S_{3}$ ). We would use the Graphene Hamiltonian to find out $M_{1}, M_{2}$ and $M_{3}$. We can express $k_{x}, k_{y}$ the components of the wave vector $\vec{k}$, in terms of $k_{1}, k_{2}$ and $k_{3}$ in the following way,

$$
k_{x}=\frac{\sqrt{3}}{2}\left(k_{2}-k_{3}\right) \text { and } k_{y}=-k_{1}+\frac{1}{2}\left(k_{2}+k_{3}\right) .
$$

So close to a lattice point, the Graphene Hamiltonian can be written as

$$
\begin{aligned}
H_{x y} & =\left(k_{x} \sigma_{x}+k_{y} \sigma_{y}\right) \otimes|x, y\rangle\langle x, y| \\
& =\left(\left(\begin{array}{cc}
0 & i k_{1} \\
-i k_{1} & 0
\end{array}\right)+\left(\begin{array}{cc}
0 & i \omega^{2} k_{2} \\
-i \omega k_{2} & 0
\end{array}\right)+\left(\begin{array}{cc}
0 & i \omega k_{3} \\
-i \omega^{2} k_{3} & 0
\end{array}\right)\right) \otimes|x, y\rangle\langle x, y| .
\end{aligned}
$$

where, $\omega=\frac{-1+\sqrt{3} i}{2}, i=\sqrt{-1}$. Since the above form of the Hamiltonian is valid close to the lattice point, i.e. within a very small region around a lattice point, it would represent infinitesimal time evolution of the quantum particle near the lattice point. This Hamiltonian shifts the chirality of the particle and translates it infinitesimally. The Hamiltonian for a position away from the lattice point is given by $H_{T}$, the translational Hamiltonian. So first the Graphene Hamiltonian acts and leave the particle slightly away from the lattice point. After that the translation operator takes it to the next lattice point. Now following Eq.(26) and neglecting the subscript in $H_{x y}$, we can write,

$$
\begin{aligned}
-i H= & -i\left[\left(\begin{array}{cc}
0 & i k_{1} \\
-i k_{1} & 0
\end{array}\right)+\left(\begin{array}{cc}
0 & i \omega^{2} k_{2} \\
-i \omega k_{2} & 0
\end{array}\right)+\left(\begin{array}{cc}
0 & i \omega k_{3} \\
-i \omega^{2} k_{3} & 0
\end{array}\right)\right] \otimes\left|n_{1}, n_{2}, n_{3}\right\rangle\left\langle n_{1}, n_{2}, n_{3}\right| \\
= & -i\left[\left(\begin{array}{cc}
0 & 1+i k_{1} \\
1-i k_{1} & 0
\end{array}\right)+\left(\begin{array}{cc}
0 & \omega^{2}\left(1+i k_{2}\right) \\
\omega\left(1-i k_{2}\right) & 0
\end{array}\right)+\left(\begin{array}{cc}
0 & \omega\left(1+i k_{3}\right) \\
\omega^{2}\left(1-i k_{3}\right) & 0
\end{array}\right)\right. \\
& \left.+i\left(\begin{array}{cc}
0 & 1+\omega+\omega^{2} \\
1+\omega+\omega^{2} & 0
\end{array}\right)\right] \otimes\left|n_{1}, n_{2}, n_{3}\right\rangle\left\langle n_{1}, n_{2}, n_{3}\right| . \\
\Rightarrow \mathcal{I}-i H \Delta t= & -i\left[\begin{array}{cc}
0 & 1+i k_{1} \Delta x_{1} \\
1-i k_{1} \Delta x_{1} & 0
\end{array}\right)+\left(\begin{array}{cc}
0 & \omega^{2}\left(1+i k_{2} \Delta x_{2}\right) \\
\omega\left(1-i k_{2} \Delta x_{2}\right) & 0
\end{array}\right) \\
& \left.+\left(\begin{array}{cc}
0 & \omega\left(1+i k_{3} \Delta x_{3}\right) \\
\omega^{2}\left(1-i k_{3} \Delta x_{3}\right) & 0
\end{array}\right)\right] \otimes\left|n_{1}, n_{2}, n_{3}\right\rangle\left\langle n_{1}, n_{2}, n_{3}\right|+I .
\end{aligned}
$$


Neglecting the identity operator above (which is not involved in any physical operations) we get three infinitesimal time evolution operators as

$$
\begin{aligned}
& W_{1}\left(n_{1}, n_{2}, n_{3}\right)=\left(\begin{array}{cc}
0 & \left|n_{1}-\Delta x_{1}, n_{2}, n_{3}\right\rangle\left\langle n_{1}, n_{2}, n_{3}\right| \\
\left|n_{1}+\Delta x_{1}, n_{2}, n_{3}\right\rangle\left\langle n_{1}, n_{2}, n_{3}\right| & 0
\end{array}\right), \\
& W_{2}\left(n_{1}, n_{2}, n_{3}\right)=\left(\begin{array}{cc}
0 & \omega^{2}\left|n_{1}, n_{2}-\Delta x_{2}, n_{3}\right\rangle\left\langle n_{1}, n_{2}, n_{3}\right| \\
\omega\left|n_{1}, n_{2}+\Delta x_{2}, n_{3}\right\rangle\left\langle n_{1}, n_{2}, n_{3}\right| & 0
\end{array}\right), \\
& W_{3}\left(n_{1}, n_{2}, n_{3}\right)=\left(\begin{array}{cc}
0 & \omega\left|n_{1}, n_{2}, n_{3}-\Delta x_{3}\right\rangle\left\langle n_{1}, n_{2}, n_{3}\right| \\
\omega^{2}\left|n_{1}, n_{2}, n_{3}+\Delta x_{3}\right\rangle\left\langle n_{1}, n_{2}, n_{3}\right| & 0
\end{array}\right) .
\end{aligned}
$$

In the previous equations we have assumed that the $-i$ can be absorbed in the normalization of the initial chiral state of the particle. More over the $\Delta x_{i}$ 's which appear are assumed to be very small displacements around the lattice points, much smaller than the lattice spacings. As the particle is $\Delta x_{i}$ distance away from a typical lattice point the Hamiltonian is governed by the term $H_{T}$, which is obtained from the translational operator $T^{\prime}\left(n_{1} \pm 1, n_{1} \pm \Delta x_{1} ; n_{2} \pm 1, n_{2} \pm \Delta x_{2} ; n_{3} \pm 1, n_{3} \pm \Delta x_{3}\right)$, responsible for translating the particle from the very neighbourhood of a lattice point to the adjacent one. When $T^{\prime}$ operates on $\left(S_{1} \otimes W_{1}+S_{2} \otimes W_{2}+S_{3} \otimes W_{3}\right)$, we can write the total time evolution operator in terms of the of an effective translation operator $T\left(n_{1}, n_{2}, n_{3}\right)$ as

$$
W\left(n_{1}, n_{2}, n_{3}\right)=T\left(n_{1}, n_{2}, n_{3}\right)\left(\left(S_{1} \otimes M_{1}+S_{2} \otimes M_{2}+S_{3} \otimes M_{3}\right) \otimes\left|n_{1}, n_{2}, n_{3}\right\rangle\left\langle n_{1}, n_{2}, n_{3}\right|\right)
$$

where,

$$
M_{1}=\left(\begin{array}{ll}
0 & 1 \\
1 & 0
\end{array}\right), M_{2}=\left(\begin{array}{cc}
0 & \omega^{2} \\
\omega & 0
\end{array}\right) \text { and } M_{3}=\left(\begin{array}{cc}
0 & \omega \\
\omega^{2} & 0
\end{array}\right)
$$

and $T\left(n_{1}, n_{2}, n_{3}\right)$ is the effective translational operator defined as,

$$
\begin{aligned}
T\left(n_{1}, n_{2}, n_{3}\right)= & \operatorname{diag}\left(\left|n_{1}-1, n_{2}, n_{3}\right\rangle,\left|n_{1}+1, n_{2}, n_{3}\right\rangle,\left|n_{1}, n_{2}-1, n_{3}\right\rangle,\left|n_{1}, n_{2}+1, n_{3}\right\rangle,\left|n_{1}, n_{2}, n_{3}-1\right\rangle,\right. \\
& \left.\left|n_{1}, n_{2}, n_{3}+1\right\rangle\right)\left\langle n_{1}, n_{2}, n_{3}\right| .
\end{aligned}
$$

Here the effective translation operator $T$ is a combination of two translations ${ }^{4}$.

\subsection{Plots for different coins}

Now for $S$, we can choose different types of mixing operators.

Case 1 : Let's take $S$ to be the three dimensional DFT coin. Therefore,

$$
S=\frac{1}{\sqrt{3}}\left(\begin{array}{ccc}
1 & 1 & 1 \\
1 & \omega & \omega^{2} \\
1 & \omega^{2} & \omega^{4}
\end{array}\right), \quad \omega=\frac{-1+\sqrt{3} i}{2}
$$

We choose our initial chiral state to be $|\chi\rangle=\frac{1}{\sqrt{3}}(|1\rangle+|2\rangle+|3\rangle) \otimes|-\rangle$. The results are shown in the following figures. The plot of Fig. 12 is generated by taking the additive time evolution operator (i. e. single step approach) and for that of 13, we take the time evolution operator in a multiplicative form (which is equivalent to a three step approach here).

In case of three step approach, we see that the probability of finding the particle collapses to a single point only. This is expected from the formulation of QRW, since for the multiplicative form of

${ }^{4}$ The translational operator is generated by two translational operators as follows:

$$
\begin{aligned}
T\left(n_{1}, n_{2}, n_{3}\right)=T^{\prime}\left(n_{1} \pm 1\right. & \left., n_{1} \pm \Delta x_{1} ; n_{2} \pm 1, n_{2} \pm \Delta x_{2} ; n_{3} \pm 1, n_{3} \pm \Delta x_{3}\right) \\
& \times T^{\prime \prime}\left(n_{1} \pm \Delta x_{1}, n_{1} ; n_{2} \pm \Delta x_{2}, n_{2} ; n_{3} \pm \Delta x_{3}, n_{3}\right)
\end{aligned}
$$

In the above expression, the $T^{\prime \prime}$ term comes from the Graphene Hamiltonian itself and the $T^{\prime}$ term comes due to putting the extra term involving $H_{T}$ into the Hamiltonian. 


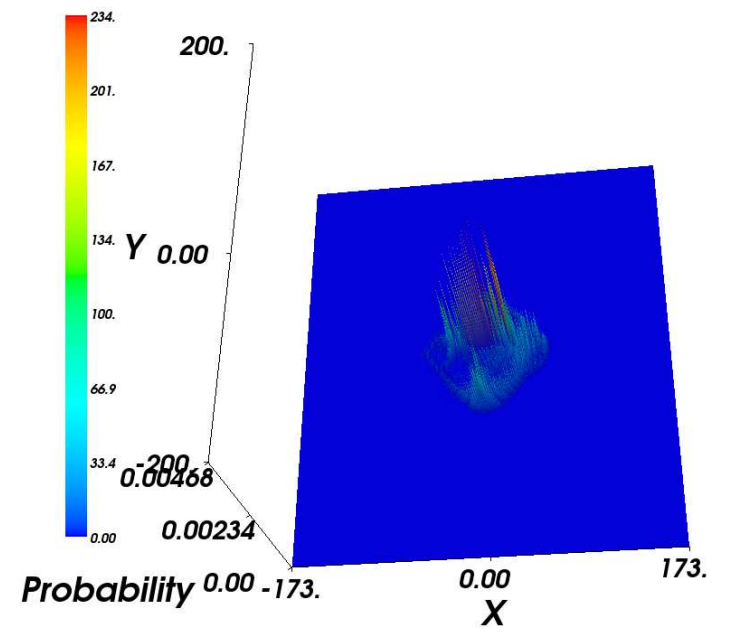

Figure 12: Plot of the probability of finding the particle after 200 time steps using the single step approach and three dimensional DFT coin of Eq.(29). The values in the color-bar are multiplied by 50000 .

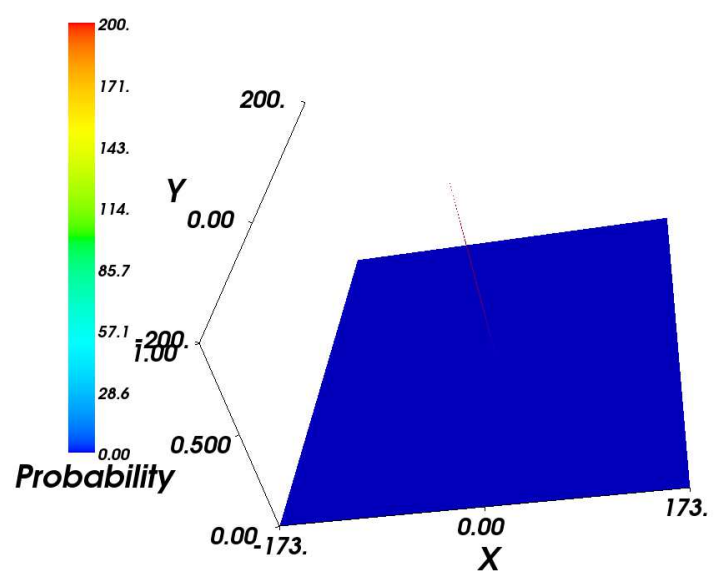

Figure 13: Plot of the probability of finding the particle after 200 time steps using the three step approach and the three dimensional DFT coin of Eq.(29). The values in the color-bar are multiplied by 200 .

the evolution operator a particle starting from origin always reaches the point $(1,-1,1)$ for every odd step and returns to the origin for every even step.

Case 2 : Now let's choose $S$ to be the three dimensional Grover coin. Therefore,

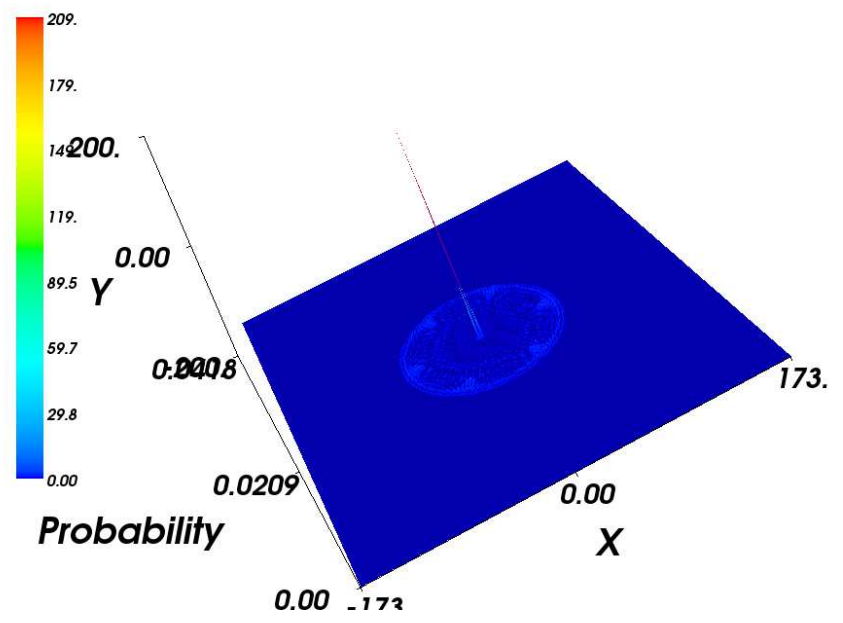

Figure 14: Plot of the probability of finding the particle after 200 time steps using the single step approach and three dimensional Grover coin of Eq.(30). The values in the color-bar are multiplied by 5000 .

$$
S=\frac{1}{3}\left(\begin{array}{ccc}
-1 & 2 & 2 \\
2 & -1 & 2 \\
2 & 2 & -1
\end{array}\right)
$$

The result for single step approach is shown in Fig. 14. Here we have chosen our initial chiral state to be $|\chi\rangle=\frac{1}{\sqrt{3}}(|1\rangle+i|2\rangle-i|3\rangle) \otimes|-\rangle$. The plot for the three step approach is same as Fig. 13 . Therefore, we omit it for the sake of brevity. 


\subsection{Calculation of variance}

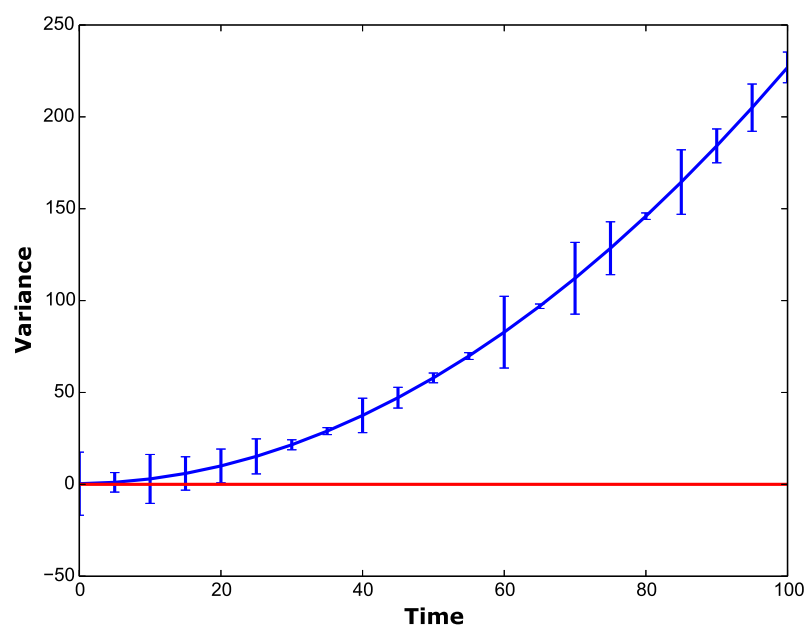

Figure 15: Plot of variance with time for the quantum walk on Graphene lattice. The blue line shows the variance for the single step approach and the red one for the three step approach. In case of the single step approach, the errorbar w.r.t. the quadratic polynomial is multiplied by 50 . Three dimensional DFT coin of Eq.(29) has been used.

We calculated the variance for different time steps for both single step and three step approach using the coins of Eq.(29) and Eq.(30). Since the results were similar, we include here only the result obtained with the three dimensional DFT coin. The plot with blue line in Fig. 15 is for evolution operator in the additive form and that of the red line in the same figure corresponds to the time evolution operator in multiplicative form. For the single step approach, the relation of variance with time has an approximated polynomial form $0.34339904+0.03773854 t+0.02227997 t^{2}$. But for the three step approach, we see that the variance is zero, which is expected since the particle always reaches a single point in this case as mentioned previously.

\section{Discussion}

Our approach to DTQRW sets constraints to the form of the Hamiltonian of the system. We found that the QW Hamiltonian can be described by the Weyl Hamiltonian with a Dirac comb type potential. In Ref. [1], though it is suggested that the Hamiltonian for QW in one, two and three dimension resembles a two component Dirac like Hamiltonian, our form of the effective Hamiltonian is distinctly different from the form of the Hamiltonian prescribed in the above reference. The other important difference with Ref. [1] for multidimensional lattices, is the way in which the evolution operator is perceived. In the referred work these evolution operators are multiplicative while in our approach the evolution operator is additive for dimensions higher than one. We have shown that the multiplicative evolution operator can be produced from our formulation also in case of two step approach by setting $k_{x}=0$ or $k_{y}=0$ at alternative lattice points.

The Fourier space formulation of QW as studied in Ref. [4] gives an analytic asymptotic form of the wave function of the particle. Hence this method results in a good understanding of the problem. In this approach, the method to solve the problem reduces to diagonalization followed by an inverse Fourier transformation. It works very well in case of $1 \mathrm{D}$ and $2 \mathrm{D}$ square lattice problem, but in case of more complicated lattice like Graphene lattice, this method becomes too complicated. In this situation, position space formulation turns out to be more useful. Position space formulation gives a formal way for numerical simulation of the problem of DTQRW for any kind of lattice.

In this article, we have calculated the variance of the probability distribution and found its quadratic dependence with number of steps. With a little modification of the standard Graphene Hamiltonian, we obtained the quantum walk on a Graphene lattice. Since the Graphene lattice is 
more constrained, we expect more centred probability distribution which is in agreement with our numerical computation of the walk as shown in Fig. 12 and Fig. 14. Due to the geometry of the Graphene lattice, $|+\rangle$ and $|-\rangle$ state can not exist for the same lattice point. So there is no interference between those states. Still in this case we find the quadratic dependence of variance with time Fig. 15. The possible reason for this quadratic dependence may be the interference between $|1\rangle,|2\rangle$ and $|3\rangle$ states. Use of multiplicative time evolution operator in this case leads to the confinement of the particle at a single point and thus no spreading occurs as seen in Fig. 13. Hence we conclude that, the additive time evolution operator is preferable over the multiplicative one.

In short, we obtained an effective Hamiltonian for QW from the evolution algorithms already existing in the literature. The form of the Hamiltonian became important when the analysis on Graphene lattice was presented, because a standard Hamiltonian of similar kind (linear in $k$ ) was already existing there. Using the similarity of the Graphene Hamiltonian and the effective Hamiltonians for QW a new algorithm of QW on Graphene lattice was presented. In a nutshell, we have presented a new and interesting way of attaining an effective Hamiltonian for QW which reproduces most of the results of earlier work in the same direction in one-dimension but differs from earlier results in higher dimensions.

\section{Acknowledgements}

N. P. acknowledges the financial support from the Council of Scientific and Industrial Research (CSIR), India as a SPM JRF.

\section{References}

[1] C. M. Chandrashekar. Two-component Dirac-like Hamiltonian for generating quantum walk on one-, two- and three-dimensional lattices. Scientific Reports, 3:2829, October 2013.

[2] Y. Aharonov, L. Davidovich, and N. Zagury. Quantum random walks. Phys. Rev. A, 48:16871690, Aug 1993.

[3] Patrici Molinàs-Mata, M. A. Muñoz, Daniel O. Martínez, and Albert-László Barabási. Ballistic random walker. Phys. Rev. E, 54:968-971, Jul 1996.

[4] A. Nayak and A. Vishwanath. Quantum Walk on the Line. eprint arXiv:quant-ph/0010117, October 2000 .

[5] Andris Ambainis, Eric Bach, Ashwin Nayak, Ashvin Vishwanath, and John Watrous. One-dimensional Quantum Walks. In Proceedings of the Thirty-third Annual ACM Symposium on Theory of Computing, STOC '01, pages 37-49, New York, NY, USA, 2001. ACM. ISBN 1-58113-349-9.

[6] J Kempe. Quantum random walks: An introductory overview. Contemporary Physics, 44(4): 307-327, 2003.

[7] Dorit Aharonov, Andris Ambainis, Julia Kempe, and Umesh Vazirani. Quantum Walks on Graphs. In Proceedings of the Thirty-third Annual ACM Symposium on Theory of Computing, STOC '01, pages 50-59. ACM, 2001.

[8] Julia Kempe. Discrete Quantum Walks Hit Exponentially Faster. Probability Theory and Related Fields, 133(2):215-235, 2005. ISSN 0178-8051.

[9] Ben Tregenna, Will Flanagan, Rik Maile, and Viv Kendon. Controlling discrete quantum walks: coins and initial states. New Journal of Physics, 5(1):83, 2003.

[10] Edward Farhi and Sam Gutmann. Quantum computation and decision trees. Phys. Rev. A, 58: 915-928, Aug 1998. 
[11] Fugao Wang and D. P. Landau. Efficient, Multiple-Range Random Walk Algorithm to Calculate the Density of States. Phys. Rev. Lett., 86:2050-2053, Mar 2001.

[12] Neil Shenvi, Julia Kempe, and K. Birgitta Whaley. Quantum random-walk search algorithm. Phys. Rev. A, 67:052307, May 2003.

[13] Andris Ambainis. Quantum Walks and Their Algorithmic Applications. International Journal of Quantum Information, 01(04):507-518, 2003.

[14] Andrew M. Childs, Richard Cleve, Enrico Deotto, Edward Farhi, Sam Gutmann, and Daniel A. Spielman. Exponential Algorithmic Speedup by a Quantum Walk. In Proceedings of the Thirtyfifth Annual ACM Symposium on Theory of Computing, STOC '03, pages 59-68. ACM, 2003.

[15] Andrew M. Childs. Universal Computation by Quantum Walk. Phys. Rev. Lett., 102:180501, May 2009.

[16] Meltem Gönülol, Ekrem Aydıner, Yutaka Shikano, and Özgür E Müstecaplıõlu. Survival probability in a one-dimensional quantum walk on a trapped lattice. New Journal of Physics, 13(3): 033037, 2011.

[17] M. Štefaňák, T. Kiss, and I. Jex. Recurrence properties of unbiased coined quantum walks on infinite $d$-dimensional lattices. Phys. Rev. A, 78:032306, Sep 2008.

[18] M Štefaňák, B Kollár, T Kiss, and I Jex. Full revivals in 2D quantum walks. Physica Scripta, 2010(T140):014035, 2010.

[19] C. A. Ryan, M. Laforest, J. C. Boileau, and R. Laflamme. Experimental implementation of a discrete-time quantum random walk on an NMR quantum-information processor. Phys. Rev. A, 72:062317, Dec 2005.

[20] Jiangfeng Du, Hui Li, Xiaodong Xu, Mingjun Shi, Jihui Wu, Xianyi Zhou, and Rongdian Han. Experimental implementation of the quantum random-walk algorithm. Phys. Rev. A, 67:042316, Apr 2003.

[21] Michał Karski, Leonid Förster, Jai-Min Choi, Andreas Steffen, Wolfgang Alt, Dieter Meschede, and Artur Widera. Quantum Walk in Position Space with Single Optically Trapped Atoms. 325 (5937):174-177, 2009.

[22] H. Schmitz, R. Matjeschk, Ch. Schneider, J. Glueckert, M. Enderlein, T. Huber, and T. Schaetz. Quantum Walk of a Trapped Ion in Phase Space. Phys. Rev. Lett., 103:090504, Aug 2009.

[23] F. Zähringer, G. Kirchmair, R. Gerritsma, E. Solano, R. Blatt, and C. F. Roos. Realization of a Quantum Walk with One and Two Trapped Ions. Phys. Rev. Lett., 104:100503, Mar 2010.

[24] A. Schreiber, K. N. Cassemiro, V. Potoček, A. Gábris, P. J. Mosley, E. Andersson, I. Jex, and Ch. Silberhorn. Photons Walking the Line: A Quantum Walk with Adjustable Coin Operations. Phys. Rev. Lett., 104:050502, Feb 2010.

[25] Alberto Peruzzo, Mirko Lobino, Jonathan C. F. Matthews, Nobuyuki Matsuda, Alberto Politi, Konstantinos Poulios, Xiao-Qi Zhou, Yoav Lahini, Nur Ismail, Kerstin Wörhoff, Yaron Bromberg, Yaron Silberberg, Mark G. Thompson, and Jeremy L. OBrien. Quantum Walks of Correlated Photons. Science, 329(5998):1500-1503, 2010.

[26] Iwo Bialynicki-Birula. Weyl, Dirac, and Maxwell equations on a lattice as unitary cellular automata. Phys. Rev. D, 49:6920-6927, Jun 1994.

[27] A. H. Castro Neto, F. Guinea, N. M. R. Peres, K. S. Novoselov, and A. K. Geim. The electronic properties of graphene. Rev. Mod. Phys., 81:109-162, Jan 2009. 


\section{Appendix A Derivation of Hamiltonian from Evolution operators in $1 \mathrm{D}$}

\section{A.1 Derivation of $H_{T}$}

If the Hamiltonian corresponding to $T$ is defined as $H_{T} \otimes \mathbb{1}$, then it can derived as follows.

$$
\begin{aligned}
e^{-i H_{T} \Delta t \otimes \mathbb{1}} & =\sum_{x}\left(\begin{array}{cc}
|x-\Delta x\rangle\langle x| & 0 \\
0 & |x+\Delta x\rangle\langle x|
\end{array}\right) \\
& =\sum_{x}\left(\begin{array}{cc}
e^{i k \Delta x} & 0 \\
0 & e^{-i k \Delta x}
\end{array}\right)\left(\mathbb{1}_{2} \otimes|x\rangle\langle x|\right) \\
& =\left(\begin{array}{cc}
e^{i k \Delta x} & 0 \\
0 & e^{-i k \Delta x}
\end{array}\right) \quad\left(\because \sum_{x}|x\rangle\langle x|=\mathbb{1}\right) \\
\Rightarrow H_{T} \Delta t \otimes \mathbb{1} & =i \ln \left(\begin{array}{cc}
e^{i k \Delta x} & 0 \\
0 & e^{-i k \Delta x}
\end{array}\right) \\
& =-k \Delta x\left(\begin{array}{cc}
1 & 0 \\
0 & -1
\end{array}\right) \\
\Rightarrow H_{T} \otimes \mathbb{1} & =-k v \sigma_{z} \quad\left(\because v=\frac{\Delta x}{\Delta t}\right) .
\end{aligned}
$$

Thus we see that the derived Hamiltonian $H_{T} \otimes \mathbb{1}$ is Hermitian.

\section{A.2 Derivation of $H_{S}$}

If we denote the Hamiltonian governing the chirality flip as $\sum_{m} H_{S} \otimes|m\rangle\langle m|$, we can write

$$
\begin{aligned}
e^{-i \sum_{m} H_{S} \Delta \tau \otimes|m\rangle\langle m|} & =\sum_{m} S \otimes|m\rangle\langle m| \\
\Rightarrow e^{-i \sum_{m} H_{S} \Delta \tau \otimes|m\rangle\langle m|}\left(\mathbb{1}_{2} \otimes|n\rangle\right) & =\sum_{m} S \otimes|m\rangle\langle m|\left(\mathbb{1}_{2} \otimes|n\rangle\right) \\
\Rightarrow e^{-i H_{S} \Delta \tau} \otimes|n\rangle & =S \otimes|n\rangle \\
\Rightarrow H_{S} & =\frac{i}{\Delta \tau} \ln S .
\end{aligned}
$$

Now $H_{S}$ is Hermitian by construction, since every unitary operator on a Hilbert space can be written as $U=e^{i A}$ for some Hermitian A.

\section{A.3 Derivation of $\mathrm{H}(\mathrm{x})$}

The total Hamiltonian $H$ is given by,

$$
H=\sum_{n} H_{S} \otimes|n\rangle\langle n|+H_{T} \otimes \mathbb{1}
$$

Therefore,

$$
\begin{aligned}
H(x) & =\left(\mathbb{1}_{2} \otimes\langle x|\right) H\left(\mathbb{1}_{2} \otimes|\alpha\rangle\right) \quad(|\alpha\rangle \text { is some ket } \in \mathbb{R}, \text { the position space. }) \\
& =\sum_{n} H_{S} \delta(x-n)\langle n \mid \alpha\rangle+H_{T}\langle x \mid \alpha\rangle \\
& =\left(\sum_{n} H_{S} \delta(x-n)+H_{T}\right)\langle x \mid \alpha\rangle \\
\Rightarrow H(x) & =\sum_{n} H_{S} \delta(x-n)+H_{T} .
\end{aligned}
$$

Here in the $2^{\text {nd }}$ step we have used a proper unit weighting factor. 


\section{Appendix B Derivation of Evolution operator from Hamiltonian in 2D square lattice}

We have,

$$
H(n, m)=H_{T} \otimes \mathbb{1}+H_{S} \otimes|n, m\rangle\langle n, m|
$$

Now following the logic as described in Sec. 3.1.1, we can say that $\left[H_{S}, H_{T}\right] \Delta t=0$. Now,

$$
\begin{aligned}
H_{S} & =\frac{i}{\Delta \tau} \ln \left(S_{x} \otimes M_{1}+S_{y} \otimes M_{2}\right), \\
\Rightarrow e^{-i H_{S} \Delta \tau \otimes|n, m\rangle\langle n, m|}\left(\mathbb{1}_{4} \otimes|n, m\rangle\langle n, m|\right) & =\left(S_{x} \otimes M_{1}+S_{y} \otimes M_{2}\right) \otimes|n, m\rangle\langle n, m| .
\end{aligned}
$$

Again,

$$
H_{T}=-\left(k_{x} v_{x}\left(\begin{array}{ll}
1 & 0 \\
0 & 0
\end{array}\right)+k_{y} v_{y}\left(\begin{array}{ll}
0 & 0 \\
0 & 1
\end{array}\right)\right) \otimes \sigma_{z}
$$

Therefore,

$$
\begin{aligned}
e^{-i H_{T} \Delta t \otimes \mathbb{1}}\left(\mathbb{1}_{4} \otimes|x, y\rangle\langle x, y|\right) & =\left(\left(\begin{array}{cc}
1 & 0 \\
0 & 0
\end{array}\right) \otimes\left(\begin{array}{cc}
e^{i k_{x} \Delta x} & 0 \\
0 & e^{-i k_{x} \Delta x}
\end{array}\right)+\left(\begin{array}{ll}
0 & 0 \\
0 & 1
\end{array}\right) \otimes\left(\begin{array}{cc}
e^{i k_{y} \Delta y} & 0 \\
0 & e^{-i k_{y} \Delta y}
\end{array}\right)\right) \otimes|x, y\rangle\langle x, y| \\
\Rightarrow T(x, y) & =\left(\begin{array}{cccc}
e^{i k_{x} \Delta x} & 0 & 0 & 0 \\
0 & e^{-i k_{x} \Delta x} & 0 & 0 \\
0 & 0 & e^{i k_{y} \Delta y} & 0 \\
0 & 0 & 0 & e^{-i k_{y} \Delta y}
\end{array}\right) \otimes|x, y\rangle\langle x, y|
\end{aligned}
$$

Therefore, we have,

$$
W(n, m)=T(n, m)\left(\left(S_{x} \otimes M_{1}+S_{y} \otimes M_{2}\right) \otimes|n, m\rangle\langle n, m|\right) .
$$

